SCIENTIFIC LETTER

\title{
Assessment of depression following acute myocardial infarction using the Beck depression inventory
}

\author{
R P Steeds, D Bickerton, M J Smith, R Muthusamy
}

Heart 2004;90:217-218. doi: 10.1136/hrt.2003.013904

S urvivors of myocardial infarction (MI) who are depressed are at increased risk of cardiac death and morbidity than those who are not depressed. ${ }^{1}$ This adverse effect is independent of the severity of the underlying ischaemic heart disease. ${ }^{1}$ However, additional care is rarely taken of depressed patients, largely due to a failure in identification. Most studies have used time consuming interviews requiring specially trained staff that may be impractical for day to day use in the clinical setting. The Beck depression inventory (BDI) is a self report inventory which is simple, accurate at identifying depression, and has been used in the USA to indicate those with an adverse prognosis. However, prevalence of depression in the UK may be different to that in the USA when using similar methods post-MI. ${ }^{2}$ Cultural and socioeconomic differences between the USA and UK may exist to uncouple the prognostic association between an elevated BDI score and adverse prognosis. Therefore, the aims of this study were to determine the prevalence of an elevated BDI and to determine the relation between BDI score and prognosis in a UK population following MI.

\section{METHODS}

All patients aged less than 75 years consecutively hospitalised with MI to a district general hospital, between 1999 and 2000, were recruited if capable of giving written, informed consent. A specific named nurse provided a copy of the BDI (version II) for completion between the time of discharge from the coronary care unit (usually 48 hours from the time of thrombolysis) and the time of discharge from the hospital (usually 5-7 days from the time of thrombolysis). Information on cultural, social, educational background, together with medical history and details of the current admission, were collated.

Follow up reviews were performed between 2-3 months after discharge and also at six months after discharge. All patients were flagged in the UK National Health Service central register for notification to us of their death and $100 \%$ follow up data were obtained.

The cut off chosen as a lower threshold for detecting depression following MI in this study was a BDI score of 12 points based on the findings of the study by Frasure-Smith, which determined that an elevated BDI-I score $\geqslant 10$ was associated with an odds ratio of 7.82 for 18 month cardiac mortality. ${ }^{1}$ A score of 10 or more on the BDI-I is equivalent to a score of 12 or more on the BDI-II. To test for differences among patients with and without depression, Fischer's exact, unpaired $t$, and Wilcoxon-Mann-Whitney tests were used as appropriate. Survival time distributions were compared using the Peto log rank test.

\section{RESULTS}

Of the 131 patients included in the study, 62 patients $(47 \%$, $95 \%$ confidence interval (CI) $39 \%$ to $56 \%$ ) scored $\geqslant 12$ at the first in-hospital BDI assessment following MI. The mean

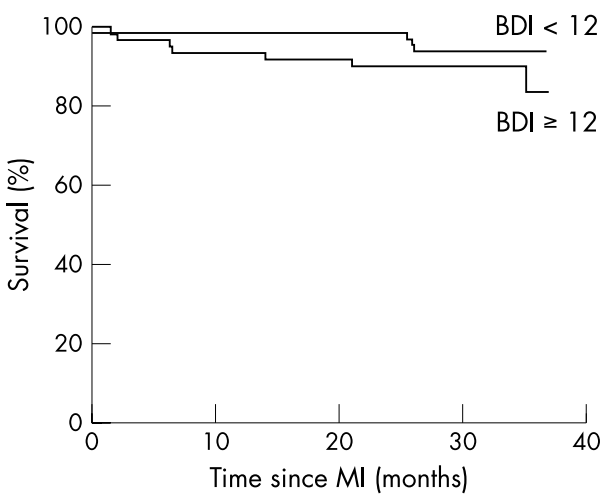

Figure 1 Survival curve for BDI scores above and below 12 .

(SD) score for those with a BDI of 12 and over at entry was 20 (7), compared to a mean score of 5 (3) for those with a BDI of below 12. There were no differences in size of MI (median creatine kinase concentration 1301 units/l (range 822-2322), location of MI (47 patients (36\%) anterior), or rate of thrombolysis (99 patients $(76 \%)$ ) between groups. There was no difference in the rate of in-hospital complications $(30$ patients $(23 \%))$. Those who had an elevated BDI at baseline were more likely to receive calcium antagonists (23 (37\%) $v 12(17 \%), \mathrm{p}=0.02)$ and less likely to receive a $\beta$ adrenoceptor antagonist on discharge (20 (32\%) $v 38$ $(55 \%), p=0.02)$, but there were no other differences in treatment following MI. No patients were discharged on antidepressant medication.

BDI scores fell in those classified as depressed, with scores at three months declining by 4.6 points ( $95 \%$ CI -6.7 to -2.4 , $\mathrm{p}<0.01$ ) and at six months by 3.3 points $(95 \%$ CI -6.0 to $-0.5, \mathrm{p}=0.02$ ). In those with a raised BDI at entry, 15 patients scored below the threshold at three months, and at six months nine patients continued to score below 12 .

Over a median follow up period of 32 months (range 25-37 months), 7 (11\%) depressed patients died compared with 4 $(6 \%)$ non-depressed patients. The estimated odds ratio (OR) for death in the depressed group compared to the nondepressed group was 1.8, although this difference was not significant (OR 1.8, 95\% CI 0.56 to $6.0 ; p=0.37$ ). Survival curves are displayed in fig 1 . The estimated odds ratio for death in the depressed group on all three visits, compared to those who were not depressed at any time, was 2.3, but this difference was not significant (OR 2.3, 95\% CI 0.42 to 15.1 , $\mathrm{p}=0.42$ ).

Abbreviations: $\mathrm{BDI}, \mathrm{Beck}$ depression inventory; $\mathrm{Cl}$, confidence interval; $\mathrm{Ml}$, myocardial infarction; OR, odds ratio 


\section{DISCUSSION}

Depression post-MI is common in the UK and is detected in $47 \%$ of patients using the BDI-II, a rate similar to that found in the USA using structured interviews by trained staff. ${ }^{2}$ This depressive symptomatology is independent of the severity of underlying disease. Prevalence of depression does fall slightly over six months, although a significant number remain affected. A trend towards adverse outcome in depressed patients was found in our study, but this was not significant. In the USA, a BDI-I score greater than 10 measured seven days post-MI was associated with an odds ratio for increased mortality at 18 months of 7.82 (95\% CI 2.42 to 25.26$).{ }^{1}$ Our study has over $90 \%$ power at $5 \%$ significance to detect such a difference in mortality. The only previous study in the UK using the BDI also failed to find a significant association with mortality at 12 months post-MI. ${ }^{4}$ This failure to find such a positive association could be due either to a reduction in the applicability of the BDI or to a reduction in the power of the link between depression and prognosis in post-MI patients in the UK. Although our study cannot exclude a smaller effect on prognosis than that previously found in the USA, the recovery of a high proportion of survivors of MI in the UK is blighted by significant psychological debility. The BDI may be used to identify these subjects who may then benefit from the safe, effective treatment for depression that is available. $^{5}$

\section{Authors' affiliations}

R P Steeds, Queen Elizabeth Hospital, Edgbaston, Birmingham, UK D Bickerton, M J Smith, R Muthusamy, Rotherham District General Hospital, Rotherham, UK

Correspondence to: Dr Richard P Steeds, Queen Elizabeth Hospital, Metchley Park Road, Edgbaston, Birmingham B15 2TH, UK;

rick.steeds@uhb.nhs.uk

Accepted 25 September 2003

\section{REFERENCES}

1 Frasure-Smith N, Lesperance F, Talajic M. Depression and 18-month prognosis after myocardial infarction. Circulation 1995;91:999-1005.

2 Schleifer SJ, Macari-Hinson MM, Coyle DA, et al. The nature and course of depression following myocardial infarction. Arch Intern Med 1989;149:1785-9.

3 Lloyd GG, Cawley RH. Psychiatric morbidity in men one week after first acute myocardial infarction. BMJ 1978;2:1453-4.

4 Lane D, Carroll D, Ring C, et al. Do depression and anxiety predict recurrent coronary events 12 months after myocardial infarction? QJM 2000;93:739-44.

5 Glassman AH, O'Connor CM, Califf RM, et al. Sertraline treatment of major depression in patients with acute $\mathrm{Ml}$ or unstable angina. JAMA 2002;288:701-9.

\section{IMAGES IN CARDIOLOGY}

\section{Secretion of atrial natriuretic peptide in idiopathic giant atrium}

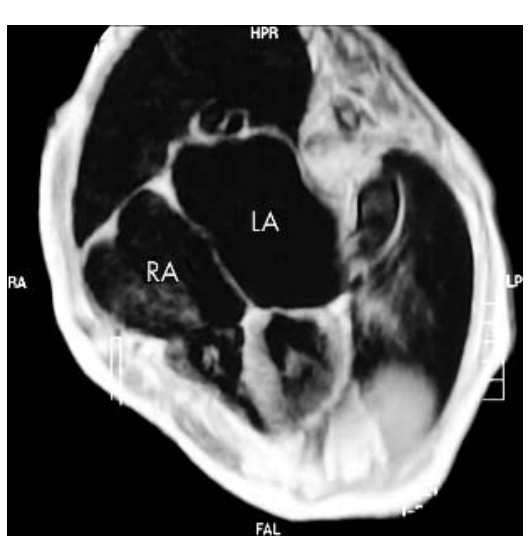

A case of idiopathic giant atrium is described. The patient died of cardiac cachexia at the $\triangle$ age of 80 years. Although she was diagnosed as having atrial fibrillation and cardiomegaly at the age of 40 years, she had not complained of any symptoms and did not receive any medical treatment. Echocardiograms and magnetic resonance imaging (upper panel) revealed pronounced bilateral atrial dilatation and normal wall motion of both ventricles. There were no structural abnormalities of the atrioventricular valves. In the compensated state of congestive heart failure, the plasma concentration of atrial natriuretic peptide (ANP) was slightly raised at $64 \mathrm{pg} / \mathrm{ml}$ (normal $<40 \mathrm{pg} / \mathrm{ml}$ ). Follow up echocardiograms showed that the dimensions of both atria increased gradually, but there were no significant changes in ejection fraction, or left ventricular dimension and wall thickness. Necropsy showed pronounced dilatation of both atria without abnormal findings in the ventricles. Microscopic findings showed degeneration of myocardial fibres without infiltration of lymphocytes, with only a few myocytes remaining in both atria. Staining with Azan revealed remarkable fibrosis in both atria, but no significant abnormalities in the ventricular walls. Staining with an anti-ANP antibody revealed no ANP containing cells in both ventricles, but notable staining in both atria (lower panel). In idiopathic giant atrium, although the atrial myocytes retain their capacity for ANP secretion, the myocytes are replaced with fibrous tissue and the amount producing ANP are attenuated.

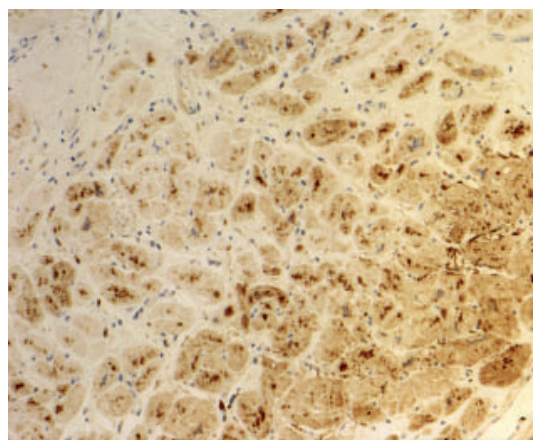

M Arima 